\title{
Time-dependent changes in paw carrageenan-induced inflammation above and below the level of low thoracic spinal cord injury in rats
}

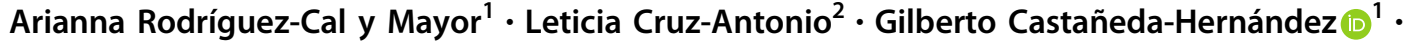 \\ Liliana Favari-Perozzi ${ }^{1} \cdot$ Gabriel Guízar-Sahagún $\mathbb{D}^{3,4}$
}

Received: 24 January 2018 / Revised: 14 March 2018 / Accepted: 23 March 2018 / Published online: 23 May 2018

(C) International Spinal Cord Society 2018

\begin{abstract}
Study design This was an animal study.

Objectives Local inflammation is attenuated below high thoracic SCI, where innervation of major lymphoid organs is involved. However, whether inflammatory responses are affected after low thoracic SCI, remains undetermined. The aim of this study was to characterize the influence of low thoracic SCI on carrageenan-induced paw swelling in intact and paralyzed limbs, at acute and subacute stages.

Setting University and hospital-based research center, Mexico City, Mexico.

Methods Rats received a severe contusive SCI at T9 spinal level or sham injury. Then, 1 and 15 days after lesion, carrageenan or vehicle was subcutaneously injected in forelimb and hindlimb paws. Paw swelling was measured over a 6-h period using a plethysmometer.

Results Swelling increased progressively reaching the maximum $6 \mathrm{~h}$ post-carrageenan injection. Swelling increase in shaminjured rats was approximately $130 \%$ and $70 \%$ compared with baseline values of forelimbs and hindlimbs, respectively. Paws injected with saline exhibited no measurable swelling. Carrageenan-induced paw swelling 1-day post-SCI was suppressed in both intact and paralyzed limbs. Fifteen days post-injury, the swelling response to carrageenan was completely reestablished in forelimbs, whereas in hindlimbs it remained significantly attenuated compared with sham-injured rats.

Conclusions SCI at low spinal level affects the induced swelling response in a different way depending on both, the neurological status of challenged regions and the stage of injury. These findings suggest that neurological compromise of the main immunological organs is not a prerequisite for the local swelling response to be affected after injury.
\end{abstract}

Electronic supplementary material The online version of this article (https://doi.org/10.1038/s41393-018-0144-5) contains supplementary material, which is available to authorized users.

Gabriel Guízar-Sahagún

guizarg@gmail.com

1 Departament of Pharmacology, Centro de Investigación y de Estudios Avanzados del Instituto Politécnico Nacional, Mexico City, Mexico

2 Departament of Pharmacy, Facultad de Estudios Superiores Zaragoza, Universidad Nacional Autónoma de México, Mexico City, Mexico

3 Research Unit for Neurological Diseases, Hospital de Especialidades Centro Médico Nacional Siglo XXI, IMSS, Mexico City, Mexico

4 Department of Experimental Surgery, Proyecto Camina A.C., Mexico City, Mexico

\section{Introduction}

Spinal cord injury (SCI) represents a considerable medical and social challenge. Beyond physical disability produced by motor and sensory impairment, SCI causes systemic and metabolic disturbances that may lead to complications that interfere, among other, with rehabilitation, social reintegration, and pharmacological treatments. Some of these complications can even be life threatening [1]. Changes related with dysfunction of the cardiovascular, respiratory, gastrointestinal, renal, endocrine, and immune systems are some of the consequences of disconnection of the spinal autonomic centers from the brain. These alterations are largely determined by the cranio-caudal level and completeness of the injury, and can occur at any time after SCI [1-3].

The immune/inflammatory dysfunction that occurs after SCI can significantly affect homeostasis [3]. An expression 
of this dysfunction is the SCI-induced immune depression syndrome (SCI-IDS), a condition characterized by impaired innate and adaptive immunity [4]. As result of SCI-IDS, patients display increased incidence and severity of systemic infections, including threatening infections such as urosepsis, pneumonia, and septicemia [5, 6].

Another potential consequence of post-injury immune/ inflammatory dysfunction is the systemic inflammatory response syndrome (SIRS), a threatening condition characterized by production of proinflammatory mediators, activation of resident immune cells, and infiltration of inflammatory cells into multiple organs such as lungs, kidneys, and liver, which can contribute to their failure $[3,7]$.

The inflammatory response depends not only on the lymphoid tissues function, but also on neurogenic mechanisms controlled in part through the release of neuropeptides such as substance $\mathrm{P}$ and calcitonin gen-related peptide from peripheral nerve endings [8-10].

The impact of SCI on local inflammatory responses is a critical topic that requires further investigation taking into account that persons with this kind of injury exhibit increased susceptibility to localized complications such as pressure sores and skin infection, associated with poor host defense and tissue repair [11, 12]. Indeed, inflammation is a key protective biological response of body to pathogens and other insults. The main purposes of inflammation are to eliminate the causal agent of damage, to remove cell and tissue debris, and to promote tissue repair [13].

In previous studies designed to evaluate the effect of SCI on local inflammation and immunity, lesions at high thoracic level have been used to compromise sympathetic innervation of the main lymphoid organs. Under these conditions, authors have found signs of impaired inflammation, synthesis of proinflammatory cytokines, and macrophage infiltration in response to inflammatory challenges below the level of injury [12, 14].

The effects that low thoracic SCI has on local inflammatory responses at both, paralyzed limbs (below injury) and of neurologically intact limbs (above injury), remains undetermined. To address this knowledge gap, it was hypothesized that despite a low SCI, where major lymphoid organs preserve normal sympathetic input, a significant reduction of localized swelling will take place in response to a local proinflammatory challenge. Hence, our objective was to characterize the influence of a cord contusion at T9 on carrageenan-induced paw swelling in both, paralyzed and intact limbs, at acute and subacute stages of injury.

Our data reveal that the carrageenan-induced paw swelling after low thoracic SCI is significantly influenced both by the neurological status of the region exposed to the inflammatory challenge, and by the time elapsed after injury.
Table 1 Experimental design

\begin{tabular}{|c|c|c|c|c|c|c|c|c|}
\hline \multirow{3}{*}{$\begin{array}{l}\text { Days } \\
\text { after } \\
\text { injury }\end{array}$} & \multicolumn{4}{|c|}{ Above injury level } & \multicolumn{4}{|c|}{ Below injury level } \\
\hline & \multicolumn{2}{|l|}{ Sham } & \multicolumn{2}{|l|}{ SCI } & \multicolumn{2}{|l|}{ Sham } & \multicolumn{2}{|l|}{ SCI } \\
\hline & Saline & CRG & Saline & CRG & Saline & CRG & Saline & CRG \\
\hline 1 & $6 / 6$ & $6 / 6$ & $7 / 8$ & $7 / 8$ & $6 / 6$ & $6 / 6$ & $7 / 8$ & $7 / 8$ \\
\hline 15 & $6 / 6$ & $6 / 6$ & $7 / 8$ & $6 / 8$ & $6 / 6$ & $6 / 6$ & $7 / 8$ & $6 / 8$ \\
\hline
\end{tabular}

Number of animals randomly allocated to each of the eight experimental groups, in which the evaluation was completed. Paw volume of both forelimb and hindlimb of each rat was measured in the same animal, before injecting carrageenan or vehicle, and every hour for $6 \mathrm{~h}$ thereafter

$C R G$ carrageenan, SCI spinal cord injury, Sham sham-injured rats

\section{Methods}

\section{Animals and experimental design}

Experiments were conducted in adult female SpragueDawley rats, 10-12 weeks old, and 250-280 g body weight, obtained from our institutional animal facility. The study involved the following steps: first, standardization of the method to induce paw swelling (12 rats); second, assessing the standardized proinflammatory challenge in eight groups of rats randomly subjected to low thoracic spinal cord contusion or sham injury control, at 1 and 15 days postsurgery. Measurements of forelimb and hindlimb paw volume were performed in the same animal (Table 1). Sample size was calculated by power analysis. The total of rats used in the study was 56 ( $n=6$ for each group of shaminjured rats and $n=8$ for each group of injured rats).

\section{Anesthesia, injury, cares, and humane endpoint}

For spinal surgery, animals were anesthetized with a mixture of ketamine $\left(80 \mathrm{mg} \mathrm{kg}^{-1}\right)$ and xylazine $\left(8 \mathrm{mg} \mathrm{kg}^{-1}\right)$ given intramuscularly. Testing of the corneal reflex and whether there was withdrawal from a painful stimulus of the hindlimb was used to ensure that the anesthesia was adequate. Prior to SCI, a laminectomy was performed aseptically at T9 maintaining meninges intact. A severe spinal cord contusion was then produced using the New York University impactor device by dropping onto the exposed dura the rod weighing $10 \mathrm{~g}$ from a height of $50 \mathrm{~mm}$. Finally, the wound was sutured in layers. Sham-injured animals were not laminectomized. They were only subjected to surgery of soft tissues.

After cord injury, animals were housed in individual cages with sterile sawdust as bedding, in a clean room, on a 12-h light/dark cycle, and under controlled atmospheric temperature and humidity. Animals were fed with standard rat chow and water ad libitum. Manual pressure of urinary bladders was performed twice a day. To prevent pain and 
Fig. 1 Effect of carrageenan (CRG) and saline injection in sham-injured rats. a Time course of paw volume change $(\Delta)$ with respect to baseline. Saline (open circles) vs. CRG (solid circles) forelimbs. Saline (open squares) vs. CRG (solid squares) hindlimbs. $* p<0.05$. b Area under curve (AUC) of $\Delta$ paw volume. Saline (open bars); CRG (solid bars). ${ }^{*} p<0.05$. Data represent mean $(\mathrm{SD})$
A

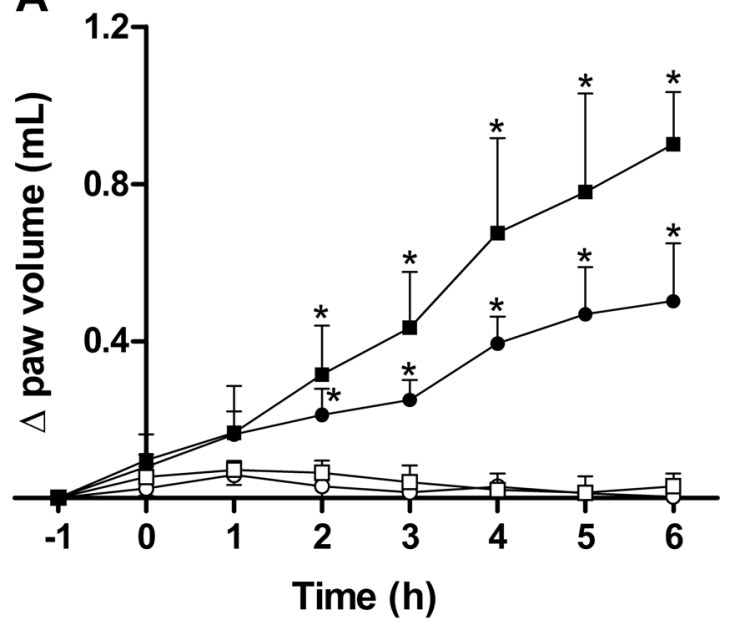

B

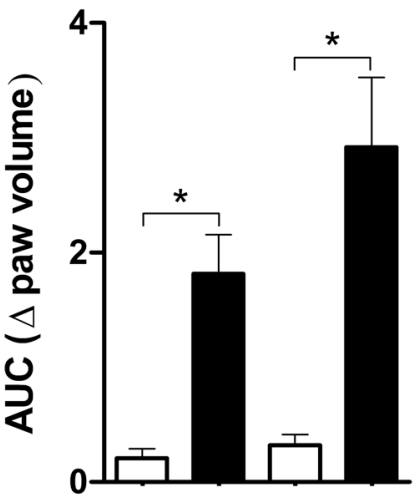

Forelimb Hindlimb self-mutilation [15], oral acetaminophen (Bristol-Myers Squibb, México) was given to all animals used in the study. Animals in short-term studies received a single dose of 30 $\mathrm{mg} \mathrm{kg}^{-1}$ when recovering from anesthesia, while animal to be evaluated 15 days post-surgery received the same dose every $12 \mathrm{~h}$, for 1 week. As prophylactic for infections, 10 $\mathrm{mg} \mathrm{kg}^{-1}$ of enrofloxacin (Parfarm, Mexico) were given orally every $24 \mathrm{~h}$ to animals in long-term studies, starting on the day of surgery and for 7 consecutive days thereafter. Euthanasia was performed: (1) at the end of an experiment; (2) in the case of life-threatening complications such as pneumonia, severe urosepsis, and abdominal evisceration due to autophagy, and (3) in the case of complications that interfere with evaluations, particularly in the case of autophagy of the limbs. Euthanasia was performed using an overdose of sodium pentobarbital $(63 \mathrm{mg})$ given IP.

\section{Induction and assessment of paw swelling}

The swelling response was induced by subcutaneous injection in the pad of left forelimb paw (above the level of injury) and hindlimb paw (below the level of injury) of 0.1 $\mathrm{mL} 2 \%$ carrageenan $\left(\lambda\right.$-carrageenan, Fluka $\left.{ }^{\circ}\right)$ diluted in saline. Injection of $0.1 \mathrm{~mL}$ of saline was used as control.

To quantify precisely paw swelling, paw volume was measured before initiation of the experiment $\left(V_{\mathrm{b}}\right)$, immediately after carrageenan or saline injection $\left(V_{0}\right)$, and 1, 2, 3, 4,5 and $6 \mathrm{~h}$ later $\left(V_{1-6}\right)$. Paw volume (in $\mathrm{mL}$ ) was determined in triplicate using a plethysmometer (Ugo Basile, Italy) [16].

\section{Data presentation and statistical analysis}

Data are presented as change $(\Delta)$ of raw data between basal paw volume $\left(V_{\mathrm{b}}\right)$ and each of subsequent $\left(V_{\mathrm{t}}\right)$ measurements $\left(\Delta\right.$ volume $\left.=V_{\mathrm{t}}(0-6 \mathrm{~h})-V_{\mathrm{b}}\right)$. Data were plotted in curves from records obtained for 6-h post-injection. Area under curve (AUC) was calculated by the trapezoidal rule for further statistical analysis.

Comparisons of paw volume changes corresponding to data plotted in curves were analyzed using two-way repeated-measures analysis of variance (ANOVA) test followed by Bonferroni's test. AUC values were compared by the Student's $t$-test.

Statistical analyses and calculation of AUC were performed using Prism V5. Differences were considered significant when $p<0.05$.

\section{Statement of ethics}

We certify that all applicable institutional and governmental regulations concerning the ethical use of animals were followed during the course of this research.

The Ethics Committee in Research of our Institution approved this study. All efforts were made to minimize animal suffering and to reduce the number of animals used. Experiments were performed in accordance with the National Institute of Health Guide for the Care and Use of Laboratory Animals.

\section{Results}

From 56 rats included in this study, 51 completed the evaluation. Two rats programed for assessment at 1-day postinjury were found dead for no apparent cause; in the case of rats to be evaluated 15 days post-injury, two were sacrificed because of pneumonia and one for autophagy of hindlimbs.

Saline injection did not produce any significant increase in paw volume. This was observed in the forelimbs and hindlimbs of both, sham-injured and SCI animals either 1 or 15 days post-lesion. 
Fig. 2 Comparison of carrageenan-induced paw swelling between injured and sham-injured rats at 1-day post-injury. Time course of paws volume change $(\Delta)$ and corresponding area under curve (AUC) in forelimbs a and hindlimbs b. SCI (triangles); Sham-injured (circles). Data represent mean (SD). ${ }^{*} p<0.05$
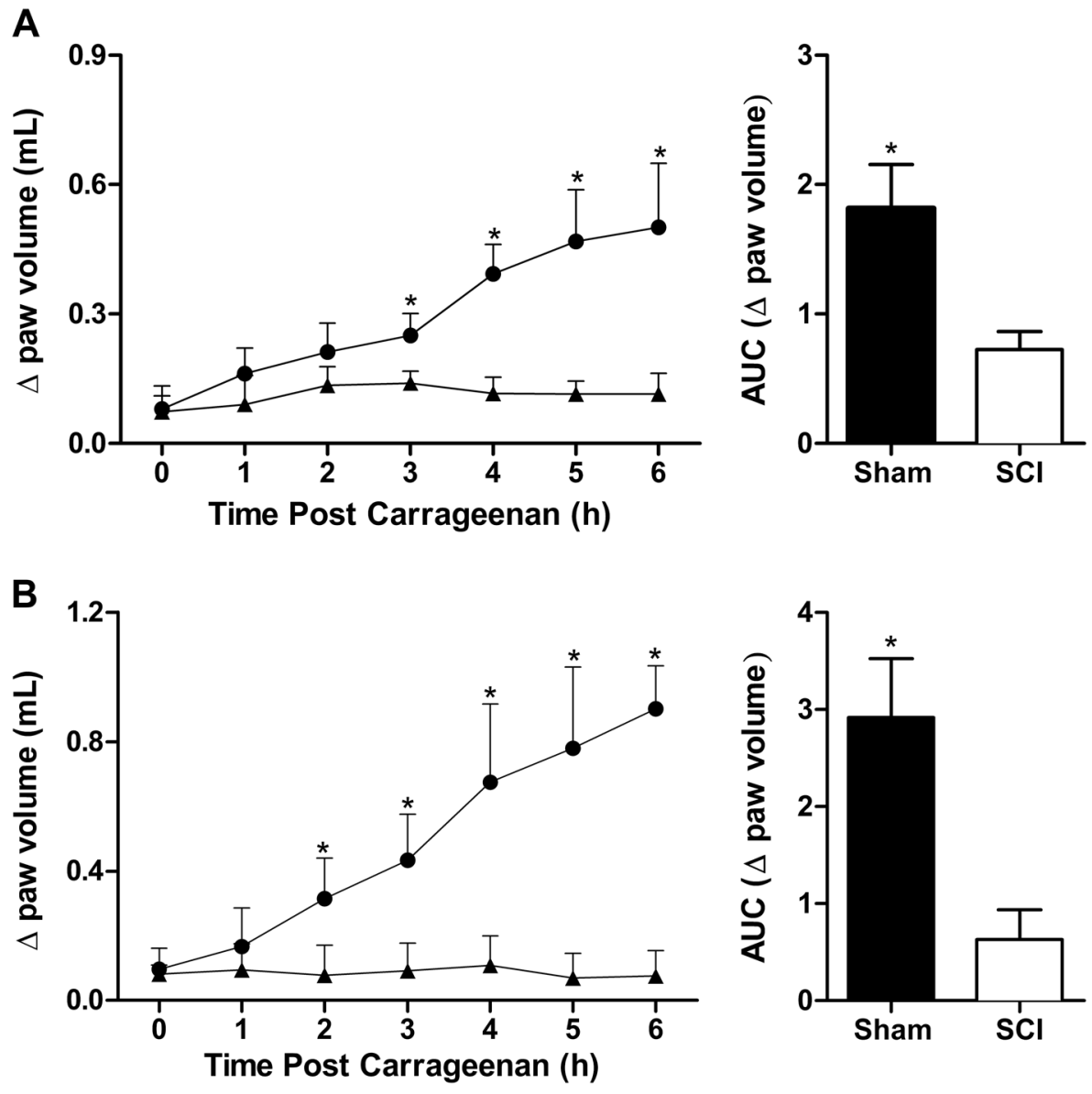

After injection of carrageenan to sham-injured rats, paw swelling increased, reaching a maximum after $6 \mathrm{~h}$. Paw increase was approximately $130 \%$ and $70 \%$ (with respect to basal measure of paws) for forelimbs and hindlimbs, respectively. The difference between saline and carrageenan achieved statistical significance at every time-point, except $1 \mathrm{~h}$ (Fig. 1a). Analysis of AUC values confirmed that carrageenan significantly increases paw volume in shaminjured rats compared with saline injection (mean $(95 \%$ confidence interval (CI)) of between-group difference: for forelimbs, $1.82(1.46-2.17)$ and $0.21(0.12-0.30 ; p<0.05)$; for hindlimbs, $2.92(2.29-3.55)$ and $0.32(0.22-0.42 ; p<$ 0.05) (Fig. 1b).

In rats subjected to SCI, the response to carrageenan injection was suppressed in the acute stage (1 day after) of injury in both, forelimbs and hindlimbs: (mean $(95 \% \mathrm{CI})$ ) of injured and sham-injured groups, $0.73(0.60-0.85)$ and 1.82 (1.46-2.17; $p<0.05$ ), for forelimbs (Fig. 2a); 0.63 $(0.35-0.91)$ and $2.92(2.29-3.55 ; p<0.05)$, for hindlimbs (Fig. 2b).

In the subacute stage (15 days after SCI), the swelling response to carrageenan was reestablished in forelimbs, as indicated by similar paw volume against time curves and a lack of significant difference when AUC values of SCI and sham-injured animals were compared: (mean (95\% CI)), 2.50 (1.82-3.18) and 1.79 (0.97-2.60), (Fig. 3a). Notwithstanding, at day 15 post-lesion, carrageenan-induced paw volume increase was impaired in hindlimbs of SCI animals, AUC being significantly lower with regard to sham-injured controls: (mean $(95 \% \mathrm{CI})$ ), $1.90(1.51-2.29)$ and 3.73 (3.12-4.33; $p<0.05)$ (Fig. 3b).

\section{Discussion}

Information on local inflammation in both paralyzed and intact regions after low thoracic SCI is scarce. Here, using the plethysmometer method to measure carrageenaninduced swelling in paws of rats with SCI at T9, we found that alterations in the swelling response depend on both, the neurological status of the challenged region and the stage of injury.

Here we assessed the proinflammatory challenge in two consecutive but differentiable phases: (1) an early stage where the animal has areflexia/hyporeflexia (spinal shock), systemic changes proper of a critical condition, and a spinal cord where the process of secondary injury predominates, and (2) an intermediate stage where reflexes have returned 
Fig. 3 Comparison of carrageenan-induced paw swelling between injured and sham-injured rats at 15 days post-injury. Time course of paws volume change $(\Delta)$ and corresponding area under curve (AUC) in forelimbs a and hindlimbs b. SCI (triangles); Sham-injured (circles). Data represent mean $(\mathrm{SD}) .{ }^{*} p<0.05$
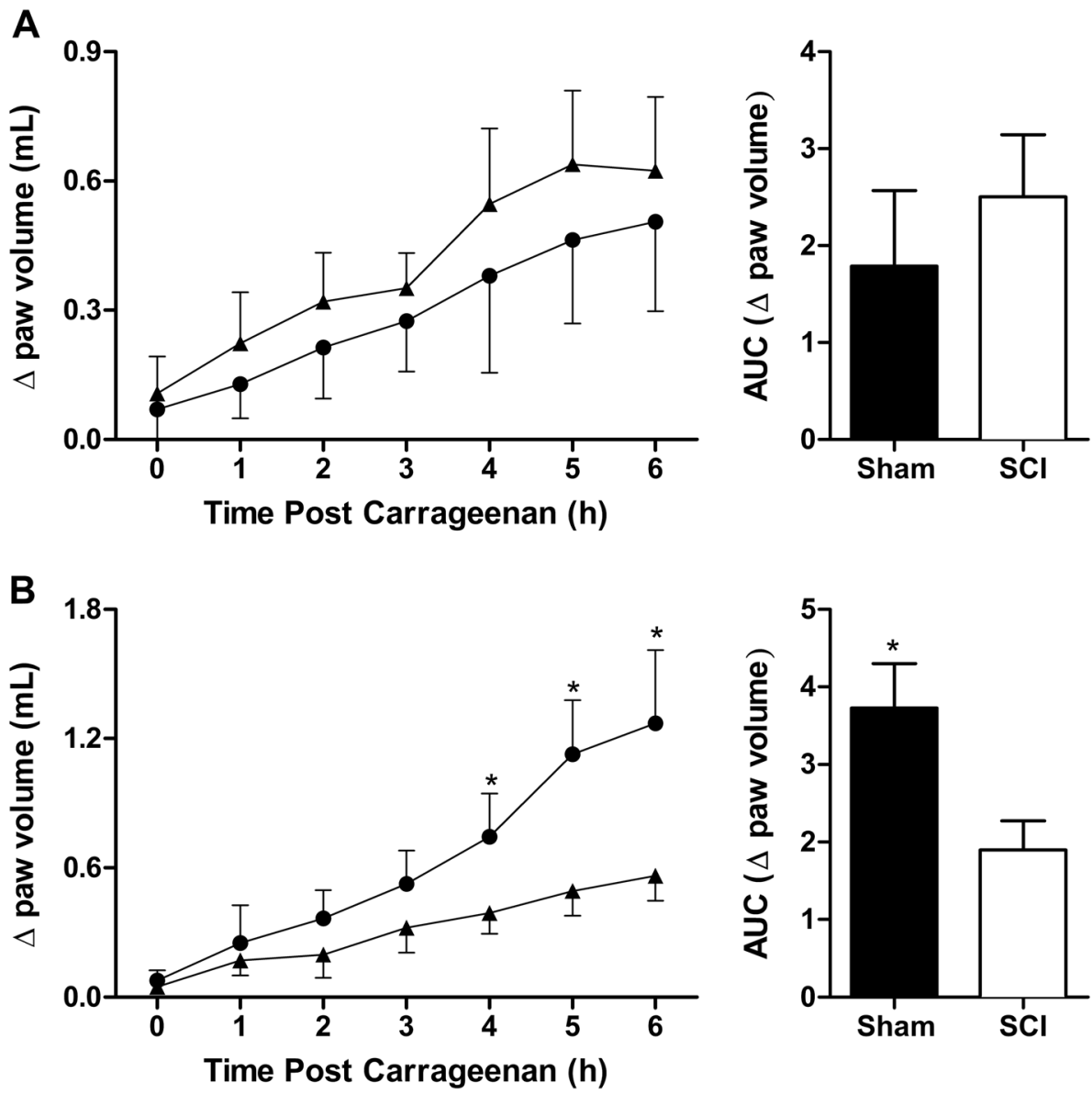

(even in the form of hyperreflexia), systemic changes tend to stabilize, and the spinal cord showing signs of remodeling [17].

\section{Rationale for using the model of carrageenan-induced inflammation paw swelling}

For the present study, the impact of SCI on local swelling was investigated using a well-characterized model, based in the subcutaneous injection of the lambda form of carrageenan. This method induces highly reproducible acute local inflammation widely used for evaluating analgesic and anti-inflammatory drugs [16, 18].

Carrageenan-induced inflammation involves both neurogenic and non-neurogenic mechanisms. In an early phase (up to $2 \mathrm{~h}$ post-injection), inflammation results from action of immediate inflammation mediators including neuropeptides (tachykinins), histamine, bradykinin, leukotrienes, and platelet-activating factor [16]. In the subsequent phase (2-6 $\mathrm{h}$ post-injection), neutrophils that migrate to sites of inflammation generate proinflammatory reactive oxygen and nitrogen species [18]. Other mediators, including prostaglandins and proinflammatory cytokines, are involved in the inflammatory response [19].

\section{Impaired paw swelling response according to post-injury stages}

Essentially, this study confirms the inhibitory effects of SCI on local inflammatory response in areas under the injury site $[12,14]$. However, we found that, at the acute stage (day 1 post-injury) the inhibitory effect on the swelling response is also present in the neurologically intact forelimb paws. Accordingly, inability of acutely injured rats to mount a swelling response, regardless of neurological status of the challenged tissue, suggest that both local and systemic mechanisms could be involved. It has been reported that neurogenic inflammation occurs after a local inflammatory insult due to antidromically conducted action potentials to the periphery [20] involving activation of the $\mathrm{Na}^{+} \mathrm{K}^{+} 2 \mathrm{Cl}^{-}$ cotransporter [21]. SCI will interrupt antidromic and thus impair the neurogenic response. On the other hand, it is known that microcirculation plays a major role in the genesis of inflammatory responses and that alterations in microcirculation can result in defective inflammatory responses [22]. Previously, we reported that early after both high and low thoracic cord injuries, microvascular blood flow is significantly decreased as a consequence of increased peripheral vascular resistance in the skin of 
forelimbs [2]. Finally, the participation of systemic mechanisms, such as hypovolemia and elevation of serum cortisol [14, 23], cannot be discarded.

Given the distinct patterns of swelling response between intact and paralyzed limbs at the subacute stage, neurological status appears to be crucial for the establishment of local inflammation. In the intact region, the swelling response to carrageenan injection is restored, possibly because mechanism such as impaired skin microcirculation [2] and the early dominant systemic anti-inflammatory effect [14] no longer occurs at this stage. On the other hand, in paralyzed regions, where the swelling response persists significantly diminished, the central autonomic pathways that regulate inflammatory/immune functions remain disrupted.

The mechanisms that explain how SCI alters inflammation at subacute stages were not studied, but several possibilities can be considered. Interruption of sensory function could be a key neural mechanism: it is known that harmful stimulation of sensory nerves results in local release of neuropeptides and other mediators that can cause vasodilation and increase capillary permeability leading to immediate signs of inflammation [10], and can also modify immune function [24]. This process, known as neurogenic inflammation, can be modulated by postganglionic sympathetic nerve fibers [8]. Presumably, as it is the case with local anesthetics, SCI attenuates neurogenic inflammation by limiting local release and axonal transport of mediators of inflammation [25]. Other possible mechanisms that could be involved in the attenuated swelling response in the subacute stage are: decrease in local inflammatory cells and cytokines immune mediators [12, 14], deficiency in the microvascular circulation induced by autonomic dysfunction [26], and morpho-functional peripheral vasculature remodeling $[27,28]$.

\section{Impact of the level of injury on paw swelling response impairment}

The available evidence suggests that alterations in immune function after SCI depend on the neurological level of lesion, being more severe the more cephalic the injury $[6,23]$.

It is known that neural modulation of immune activity depends mainly on sympathetic innervation of the lymphoid organs [29], and that a SCI occurring to the upper thoracic and cervical spine will block regulatory sympathetic input to major lymphoid organs [23,30]. Therefore, high thoracic injury levels have been used in studies designed to evaluate SCI impact on inflammation [12, 14].

Given the neurological level of injury used in the present study (T9), it could be assumed that locally induced inflammation would not be significantly affected considering that the sympathetic input of major lymphoid organs would not be disrupted. Hence, our results suggest that systemic immune depression associated with blockage of sympathetic input to major lymphoid organs is not a prerequisite to altered inflammation in regions below the site of injury.

\section{Study limitations and future research}

In the present study, we have investigated the net effect of SCI on local swelling response to a proinflammatory challenge. Nonetheless, the precise mechanisms behind the outcomes were not studied. More in depth mechanistic studies are warranted for a better understanding of how SCI at both, high and low spinal levels modify inflammatory responses. Moreover, our observations were limited to 15 days post-injury. In future studies, it would be interesting to inquire what happens in chronic stages, when the lesion tends to stabilize.

\section{Clinical relevance}

In the clinical setting, disorders of the skin and subcutaneous tissues are relatively frequent in persons with SCI; they negatively affect the quality of life and can lead to serious systemic complications [11]. Our results contribute to understand the characteristics of impaired local responses to a proinflammatory challenge at different stages after SCI. It could be predicted that attenuated inflammatory responses will result in enhanced susceptibility to infections and delayed wound healing in persons with SCI [11, 12]. It should be considered that the initial inflammatory response is a critical factor in recruiting and activating immunocompetent cells, as well as in providing the conditions for tissue repair [13].

In conclusion, the current study findings suggest that the exposure to a proinflammatory challenge to intact and paralyzed limbs of rats at acute and subacute stages of a clinically relevant model of SCI provides a pattern of the swelling response in this pathologic condition. SCI at low spinal level affects the induced swelling response in a different way depending on both, the neurological status of challenged regions and the stage of injury. These findings suggest that neurological compromise of the main immunological organs is not a prerequisite for the local swelling response to be affected after injury. The mechanisms underlying these alterations deserve further attention.

Acknowledgements We thank Angelina Martinez-Cruz for her invaluable technical assistance with spine surgery.

Funding AR-C received a fellowship grant (number 378379) from the National Council for Science and Technology (CONACYT, http://www.conacyt.mx/pci/index.php) to support her doctoral studies. 
Author contributions AR-C was responsible for design of methodology, writing the protocol, performing the experimental procedures, collecting and analyzing data, interpreting results, and writing original draft. LC-A was responsible for design of methodology, management and coordination of research activity, planning, and execution, as well as analysis and interpretation of data. GC-H was responsible for conception and design of the study, providing study materials, animals, and analysis tools, analysis and interpretation of data, writing original draft, and presentation of the critically revised work. LF-P was responsible for oversight and leadership the research activity planning and execution, including mentorship external to the core team, and analysis and interpretation of data. GG-S was responsible for conception and design of the study, review of the protocol, analysis and interpretation of data, writing original draft, and presentation of the critically revised work. All authors revised and approved the final version of the manuscript.

\section{Compliance with ethical standards}

Conflict of interest The authors declare that they have no conflict of interest.

\section{References}

1. Guizar-Sahagun G, Castaneda-Hernandez G, Garcia-Lopez P, Franco-Bourland R, Grijalva I, Madrazo I. Pathophysiological mechanisms involved in systemic and metabolic alterations secondary to spinal cord injury. Proc West Pharmacol Soc. 1998;41:237-40.

2. Guizar-Sahagun G, Velasco-Hernandez L, Martinez-Cruz A, Castaneda-Hernandez G, Bravo G, Rojas G, et al. Systemic microcirculation after complete high and low thoracic spinal cord section in rats. J Neurotrauma. 2004;21:1614-23.

3. Sun X, Jones ZB, Chen XM, Zhou L, So KF, Ren Y. Multiple organ dysfunction and systemic inflammation after spinal cord injury: a complex relationship. J Neuroinflamm. 2016;13:260.

4. Riegger T, Conrad S, Liu K, Schluesener HJ, Adibzahdeh M, Schwab JM. Spinal cord injury-induced immune depression syndrome (SCI-IDS). Eur J Neurosci. 2007;25:1743-7.

5. Cotton BA, Pryor JP, Chinwalla I, Wiebe DJ, Reilly PM, Schwab $\mathrm{CW}$. Respiratory complications and mortality risk associated with thoracic spine injury. J Trauma. 2005;59:1400-7.

6. Brommer B, Engel O, Kopp MA, Watzlawick R, Muller S, Pruss $\mathrm{H}$, et al. Spinal cord injury-induced immune deficiency syndrome enhances infection susceptibility dependent on lesion level. Brain. 2016;139:692-707.

7. Gris D, Hamilton EF, Weaver LC. The systemic inflammatory response after spinal cord injury damages lungs and kidneys. Exp Neurol. 2008;211:259-70.

8. Basbaum AI, Levine JD. The contribution of the nervous system to inflammation and inflammatory disease. Can J Physiol Pharmacol. 1991;69:647-51.

9. Lin Q, Wu J, Willis WD. Dorsal root reflexes and cutaneous neurogenic inflammation after intradermal injection of capsaicin in rats. J Neurophysiol. 1999;82:2602-11.

10. Chiu IM, von Hehn CA, Woolf CJ. Neurogenic inflammation and the peripheral nervous system in host defense and immunopathology. Nat Neurosci. 2012;15:1063-7.

11. Backhaus M, Citak M, Tilkorn DJ, Meindl R, Schildhauer TA, Fehmer T. Pressure sores significantly increase the risk of developing a Fournier's gangrene in patients with spinal cord injury. Spinal Cord. 2011;49:1143-6.

12. Marbourg JM, Bratasz A, Mo X, Popovich PG. Spinal cord injury suppresses cutaneous inflammation: implications for peripheral wound healing. J Neurotrauma. 2017;34:1149-55.

13. Reinke JM, Sorg H. Wound repair and regeneration. Eur Surg Res. 2012;49:35-43.

14. Macneil BJ, Nance DM. Skin inflammation and immunity after spinal cord injury. Neuroimmune Biol. 2001;1:459-73.

15. Young W. MASCIS spinal cord contusion model. In: Chen J, Xu $\mathrm{ZC}, \mathrm{Xu} \mathrm{XM}$, Zhang JH, editors. Animal models of acute neurological injuries.. Totowa, NJ, USA: Humana Press; 2009. p. 411-21.

16. Posadas I, Bucci M, Roviezzo F, Rossi A, Parente L, Sautebin L, et al. Carrageenan-induced mouse paw oedema is biphasic, ageweight dependent and displays differential nitric oxide cyclooxygenase-2 expression. Br J Pharmacol. 2004;142:331-8.

17. Ditunno J, Little J, Tessler A, Burns A. Spinal shock revisited: a four-phase model. Spinal Cord. 2004;42:383-95.

18. Morris CJ. Carrageenan-induced paw edema in the rat and mouse. Methods Mol Biol. 2003;225:115-21.

19. Annamalai P, Thangam EB. Local and systemic profiles of inflammatory cytokines in carrageenan-induced paw inflammation in rats. Immunol Invest. 2017;46:274-83.

20. Willis WD Jr.. Dorsal root potentials and dorsal root reflexes: a double-edged sword. Exp Brain Res. 1999;124:395-421.

21. Valencia-de Ita S, Lawand NB, Lin Q, Castaneda-Hernandez G, Willis WD. Role of the $\mathrm{Na}+-\mathrm{K}+-2 \mathrm{Cl}-$ cotransporter in the development of capsaicin-induced neurogenic inflammation. $\mathrm{J}$ Neurophysiol. 2006;95:3553-61.

22. Granger DN, Senchenkova E (eds). Inflammation and the microcirculation. In: Colloquium series on integrated systems physiology: from molecule to function. Morgan \& ClaypoolLife Sciences; San Rafael, CA, USA 2010. p. 1-87.

23. Lucin KM, Sanders VM, Jones TB, Malarkey WB, Popovich PG. Impaired antibody synthesis after spinal cord injury is level dependent and is due to sympathetic nervous system dysregulation. Exp Neurol. 2007;207:75-84.

24. Lotz M, Vaughan JH, Carson DA. Effect of neuropeptides on production of inflammatory cytokines by human monocytes. Science. 1988;241:1218-21.

25. Deruddre S, Combettes E, Estebe JP, Duranteau J, Benhamou D, Beloeil $\mathrm{H}$, et al. Effects of a bupivacaine nerve block on the axonal transport of tumor necrosis factor-alpha (TNF-alpha) in a rat model of carrageenan-induced inflammation. Brain Behav Immun. 2010;24:652-9.

26. Frisbie JH. Microvascular instability in tetraplegic patients: preliminary observations. Spinal Cord. 2004;42:290-3.

27. West C, Alyahya A, Laher I, Krassioukov A. Peripheral vascular function in spinal cord injury: a systematic review. Spinal Cord. 2013;51:10-19.

28. Phillips A, Matin N, Frias B, Zheng M, Jia M, West C, et al. Rigid and remodelled: cerebrovascular structure and function after experimental high-thoracic spinal cord transection. J Physiol. 2016;594:1677-88.

29. Mignini F, Streccioni V, Amenta F. Autonomic innervation of immune organs and neuroimmune modulation. Auton Autacoid Pharmacol. 2003;23:1-25.

30. Zhang Y, Guan Z, Reader B, Shawler T, Mandrekar-Colucci S, Huang K, et al. Autonomic dysreflexia causes chronic immune suppression after spinal cord injury. J Neurosci. 2013;33: 12970-81. 